\title{
Energy-saving and quickly-responding SMA actuator
}

\author{
K. K. Jee ${ }^{\mathrm{a}, 1}$, Y. B. Kim ${ }^{1}$, J. H. Han ${ }^{1}$, J. M. Park ${ }^{2}$ and W. Y. Jang ${ }^{3}$ \\ ${ }^{1}$ Div. of Materials Science and Engineering, Korea Institute of Science and Technology, Seoul 136-791, Korea \\ ${ }^{2}$ School of Materials Science and Engineering Hongik University, Jochiwon 339-800, Korea \\ ${ }^{3}$ Dept. of Metallurgical and Materials Engineering, Chosun University, Gwangju 501-759, Korea
}

\begin{abstract}
The main drawbacks of SMA actuators are a slow response and a waste of electric power. The power should be continuously supplied for SMA elements to remain austenite state until the actuator begins to take the other position. The problems are more serious when batteries and thick SMA elements are used. We propose a new SMA actuator which consumes little energy and responds very quickly. The actuator consists of a specially designed bias spring and two SMA elements which exert the force in opposite direction to each other. The bias spring, unlike a common spring which has one stable position, has two stable positions. A SMA1, for instance, contracts on heating by electricity, the actuator takes one stable configuration. No further power supply is necessary to maintain the stable position, providing enough time for the SMA1 to cool. When the other SMA2 is heated, it contracts with enough force to move the bias spring and to expand the already-cooled SMA1. Power supply stops immediately after the actuator takes the other stable configuration, causing power saving and quick response.
\end{abstract}

\section{Introduction}

SMA actuators are cheap, light and noiseless, thus they have been expected to replace the solenoids and motors. $(1,2)$ However, their application has not been so successful as has been expected. The main reasons are thought to be slow response and power consumption. Fig. 1 shows working principle of a conventional SMA actuator which is composed of a SMA wire and a bias coil spring. (3) When electric current flows, contraction of the SMA wire occurs, lengthening the bias spring. On power-off, the SMA wire of martensitic phase is easily extended by contraction of the bias spring.

Although the working principle is simple, the conventional SMA actuators have some demerits, making their application very limited. One of them is power waste. In order to maintain the Fig.1 (a) state, power supply must be continued to keep the SMA element above Af temperature. . If power breaks, martensitic transformation takes place, the actuator changes to (b) state. The problem of power consumption is especially serious to application to small battery-using instrument such as camera or cellular phone. Another obstacle is heating and cooling rate which relates to response. It is desirable for the SMA elements to be heated to a suitable temperature quickly and then stay. However, it is difficult to determine the proper current value whose existence is doubtful. A high current makes the SMA element overheated, while a low current cause sluggish response on heating. When power supply stops by the external signal, it is preferable for the SMA element to cool below transformation temperature quickly. However, the SMA element has been heated until the moment low temperature position is wished. There is a delay in action of the actuator as much as cooling time below Ms temperature. The trouble becomes more serious when thick SMA elements are used3), because in most cases, SMA elements are cooled by air convection. An ideal SMA actuators needs electric power only in the middle of movement, so that the high temperature position can be maintained without further power consumption. The ideal SMA actuators can solve the problems of power waste as well as sluggish response. The aim of this work is to propose the ideal SMA actuator which is composed of a peculiar bias spring and two SMA elements. The unique bias spring, unlike common springs which have one stable position, has two stable positions.

\footnotetext{
${ }^{\mathrm{a} e-m a i l ~: ~ K K k k j e e d k i s t . r e \cdot k r}$
} 
(a)

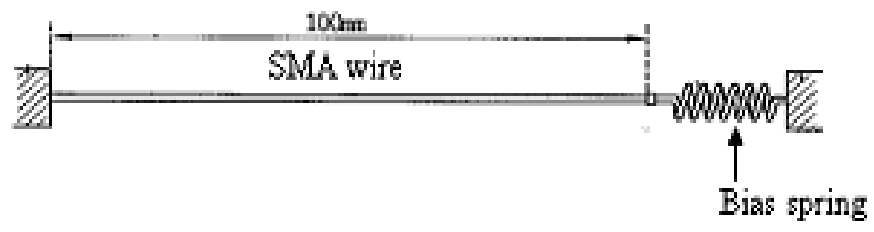

(b)

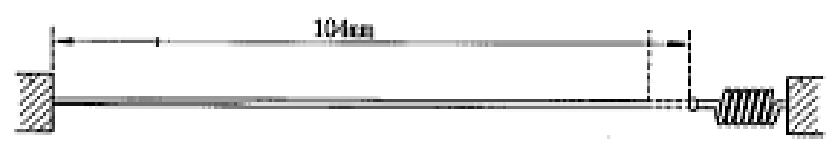

Fig. 1. Working principle of conventional SMA actuator

(a)

$$
\mathrm{A}
$$
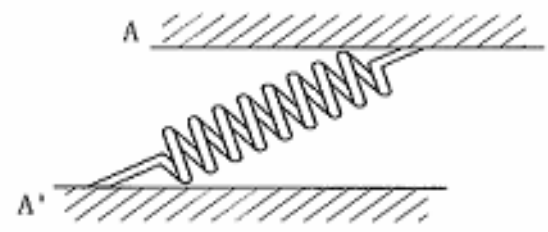

(b)

^
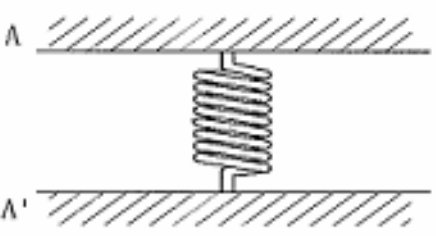

(c)

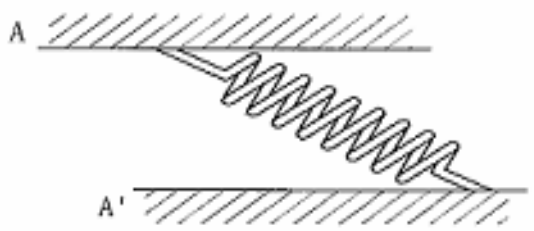

Fig. 2. Bias spring with two stable positions using compression coil spring

\section{Methodology}

Fig. 2 shows an example of structure of the bias spring which has two stable positions It consists of two parallel plates and a compression coil spring whose ends are connected to either plate. It is convenient to assume that the upper plate (A) is fixed, and the lower plate (A') is allowed to move horizontally, but not vertically. When the $\mathrm{A}^{\prime}$ plate is moved rightward, the compression stress of the spring increases with an increase in displacement. The compression stress of the spring reaches its maximum at Fig. 2(b) where the spring length becomes minimum. However, the maximum resistance of the A' plate is located near halfway between Fig. 2(a) and (b), though the exact position depends on various factors including initial compression stress. At Fig. 2(b), the A' plate can move automatically leftward or rightward according to impetus, because the movement in any direction causes reduction in compression stress. Minimum compression stress can be attained when the lower plate moves to Fig 2(a) or (c) therefore the bias structure has two stable positions. Figure 3 show another example of bias spring structure which uses a wire spring instead of compression coil spring. 


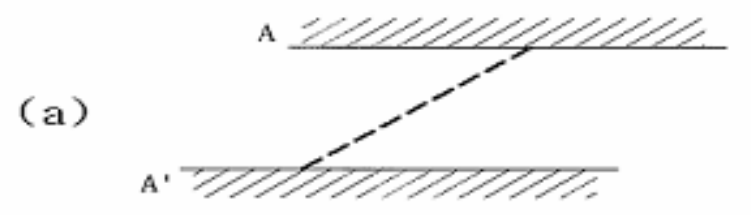

(b)

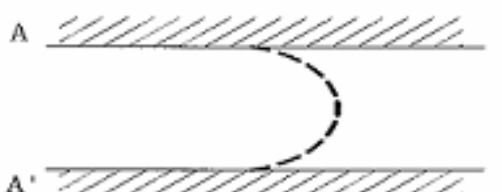

(c)

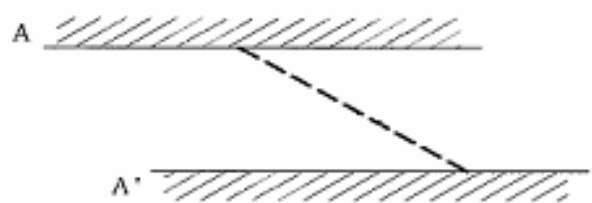

Fig. 3. Bias spring with two stable positions using wire spring

Schematically illustrated in Fig. 4 are compression force of the spring and force for the A' plate to return to (a) position vs displacement of the $\mathrm{A}^{\prime}$ plate. As the $\mathrm{A}^{\prime}$ plate is moved rightward, the spring force increases upto (b), however, the compression force decreases by further movement to (c). The force for the $A^{\prime}$ plate to return to (a) position first increases, however, decreases due to an increase in angle of the spring force and the movement direction. At the (b) position, the spring exerts no force to move the A' plate either leftward or rightward, because the spring becomes perpendicular to the movement direction. In the displacement between (a) and (b), the A' plate takes (a) position, while (c) position is stable in the displacement between (b) and (c).

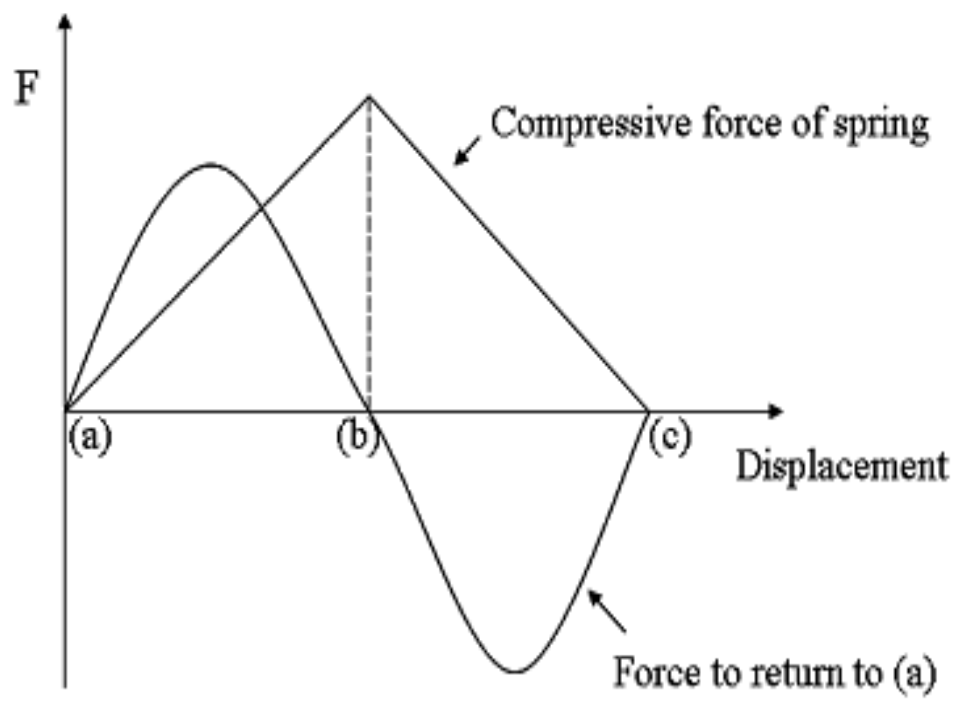

Fig. 4. Schematic illustration of compression force of the spring and force for the $\mathrm{A}^{\prime}$ plate to return to (a) position vs displacement

The ideal actuator can be made by installing two SMA elements which are tension coil springs in the bias spring structure of the wire spring as in Fig. 5. The SMA1 is set to move the lower plate rightward on heating above Af temperature while SMA2 acts in the opposite direction. In Fig. 5(a), the SMA1 has expanded, while the SMA2 has contracted and the bias spring is in the stable state. The SMA1 and SMA2 elements exert a small force, because they possess martensitic phase. 
(a)

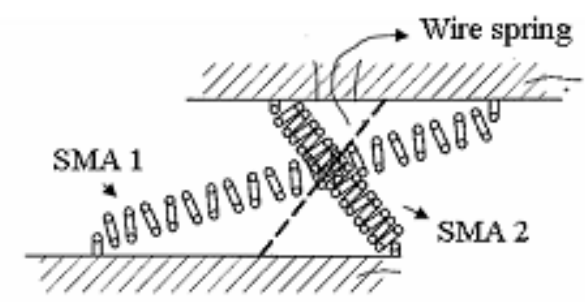

(b)
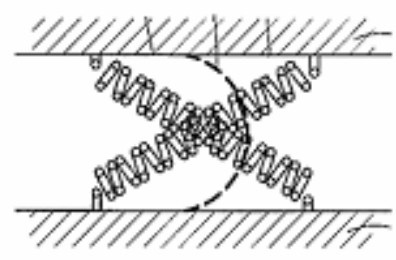

(c)

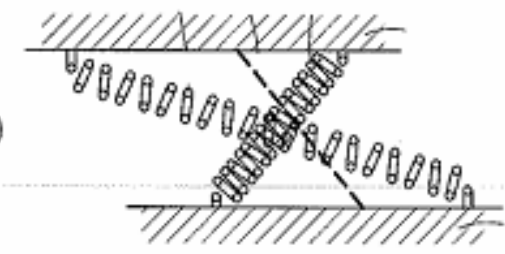

Fig. 5. SMA actuator consisting of two SMA elements bias spring using wire spring

When electric power is supplied to the SMA1, which contracts with enough force to overcome resistance of bias spring and to extend SMA2 of low temperature phase. Once the lower plate reached the Fig. 5(b), the bias helps rather than hinders the working of SMA1. Expansion of SMA2 needs a little force, since it possess low temperature phase. When the lower plate move past Fig. 5(b), power supply stops, thus the SMA1 begins to cool. The actuator takes the position of Fig. 5(c) for a while before the SMA2 starts working. The contraction force must outdo force of the bias spring as well as extend the SMA1. As the power for SMA1 has been off for a while, SMA1 is easily deformed.

According to simple experiment, it takes about $1 \mathrm{sec}$ to heat a Ni-Ti coil with a wire dia. of $0.3 \mathrm{~mm}$ and a coil dia. of $3.0 \mathrm{~mm}$ from room temperature to $80^{\circ} \mathrm{C}$ (above Af) by supplying a current of 1.8-2.0A. About $15 \mathrm{sec}$ is taken for the coil to cool to room temperature (below Ms).

If a conventional SMA actuator, for instance, is designed so that the SMA element is repetitively powered for 5 min. and not powered for $10 \mathrm{~min}$, and the actuator has worked for $1 \mathrm{hr}$. The electric power has been supplied for $20 \mathrm{~min}$. However, for the new actuator, total time of power supply is less than 10sec. As for response, we have to wait $10 \mathrm{sec}$ for the conventional SMA actuator to work on cooling. The new SMA actuator is expected to have no delay in action. The newly proposed SMA actuator is under construction.

\section{Summary}

A new SMA actuator which needs little electricity and responds very quickly is proposed in this work. The actuator consists of a specially designed bias spring and two SMA elements. The bias spring has two stable positions. SMA element once moves the position of the bias spring to the other, no further power supply is necessary to maintain the stable position, providing enough time for the SMA element to cool. Therefore economical actuator with quick response can be accomplished.

\section{References}

[1] Francesco Butera, Advanced Materials \& Processes/ March 2008/ 37-40

[2] I. Ohkata, Y. Sizuki, in: k. Otsuka, C. M. Wayman (Eds.), Shape Memory Materials, Cambridge University

Press, Cambridge, 1998, p. 240

[3] Technical information from Dynalloy, Inc 